\title{
Asynchronous data assimilation with the EnKF
}

\author{
By PAVEL SAKOV ${ }^{1 *}$, GEIR EVENSEN ${ }^{1,2}$ and LAURENT BERTINO ${ }^{1},{ }^{1}$ Nansen Environmental and \\ Remote Sensing Center, Thorm $\phi$ hlensgate 47, Bergen 5006, Norway; ${ }^{2}$ Statoil Research Center, Sandsliveien 90 , \\ Bergen 5020, Norway
}

(Manuscript received 6 May 2009; in final form 1 October 2009)

\begin{abstract}
This study revisits the problem of assimilation of asynchronous observations, or four-dimensional data assimilation, with the ensemble Kalman filter (EnKF). We show that for a system with perfect model and linear dynamics the ensemble Kalman smoother (EnKS) provides a simple and efficient solution for the problem: one just needs to use the ensemble observations (that is, the forecast observations for each ensemble member) from the time of observation during the update, for each assimilated observation. This recipe can be used for assimilating both past and future data; in the context of assimilating generic asynchronous observations we refer to it as the asynchronous EnKF. The asynchronous EnKF is essentially equivalent to the four-dimensional variational data assimilation (4D-Var). It requires only one forward integration of the system to obtain and store the data necessary for the analysis, and therefore is feasible for large-scale applications. Unlike 4D-Var, the asynchronous EnKF requires no tangent linear or adjoint model.
\end{abstract}

\section{Introduction}

We consider the problem of assimilation of observations made at the time different to the time of the update, or asynchronous observations, with the ensemble Kalman filter (EnKF, Evensen, 1994). This problem is commonly referred to as 'four-dimensional' (4-D) data assimilation, in contrast to 'threedimensional' (3-D) data assimilation, when all assimilated observations are assumed to be synchronous, or made at the time of the update.

The problem of assimilation of asynchronous observations with ensemble methods has been investigated in a number of studies (e.g. van Leeuwen and Evensen, 1996). Evensen and van Leeuwen (2000) provided a detailed formulation of the problem in a probabilistic framework and introduced a modification of the EnKF called ensemble Kalman smoother (EnKS). The practical aspects of the EnKS have been further considered in Evensen (2003) and Evensen (2007). Independently, the problem has been studied by Hunt et al. (2004), who developed a method called 4D-EnKF. Later, a simpler method called 4D-LETKF has been used in Hunt et al. (2007).

The rationale for this work is two-fold. First, we would like to provide a coherent view on the problem of assimilation of asynchronous observations with EnKF, which, we believe, may be useful, considering the diversity of the literature on the subject.

\footnotetext{
*Corresponding author. e-mail: pavel.sakov@nersc.no DOI: $10.1111 / \mathrm{j} .1600-0870.2009 .00417 . x$
}

We will show that the EnKS provides the necessary solution. To avoid a possible confusion and underline that the method can be used both as a filter, assimilating past asynchronous data, and as a smoother, assimilating future data, we call it asynchronous EnKF. Second, we would like to point that while the asynchronous EnKF and the 4D-EnKF are mainly equivalent, the 4D-EnKF represents an unnecessary and potentially vulnerable complication of the asynchronous EnKF. Unlike the 4D-EnKF, the 4D-LETKF is essentially equivalent to the asynchronous EnKF.

A straightforward way to handle observations scattered in time with the EnKF is to assimilate each batch of observations at the model time equal to the observation time. Unfortunately, this approach is not feasible for large-scale forecasting applications because each assimilation requires a disruption of the ensemble integration, an update of the ensemble, and a restart. These operations require a certain amount of time in a given operational setting, and can become too expensive if performed frequently. It is therefore preferable to have a longer assimilation window, assimilating all collected observations in a single update and taking into account the evolution of the state and state error covariance over the length of the window.

The problem of 4-D assimilation also arises in reanalysis, when it is often desirable to improve the EnKF analysis by taking advantage of future observations. Data assimilation of future and past as well as present observations is commonly referred to as the smoothing problem. The EnKS (Evensen and van Leeuwen, 2000) is, similarly to the EnKF, a sequential method; that is, observations can be assimilated sequentially during a 
forward integration. Evensen (2003) observed that the ensemble transform matrix in the EnKF at a given time can be used for updating the smoother estimate at any previous time. It is important for practice that the final transform matrix is 'only dependent on the ensemble' at the observation time, and 'then only at measurement locations' (Evensen, 2007, p. 129, p. 130, eq. 9.43). In other words, for an update with the EnKS it is sufficient to know only the ensemble observations that can be stored during a forward run of the ensemble, rather than the full ensemble at each observation time.

In this work, we consider assimilation of asynchronous data with the EnKF by using a simple concept of propagation of corrections along the forecast system trajectory. Essentially, the necessary modifications to the EnKF involve the use of ensemble observations from the time of observations during the update. The use of ensemble observations from the time of observations makes the method equivalent to the EnKS as presented in Evensen (2003); however, we decided to refer to the method in this work as the asynchronous EnKF, or AEnKF, for two reasons. First, the acronym EnKS is historically identified with a specific smoother solution, and could lead to confusion when referred to a generic method. Second, we would like to underline that the asynchronous EnKF represents a very simple, almost trivial modification of the EnKF, rather than a new method. We use the acronym AEnKF in this paper only to distinguish it from the standard (synchronous) EnKF; elsewhere the method can perhaps be simply referred to as the EnKF, once it is made clear that it is used for assimilating asynchronous observations.

The outline of this work is as follows. Section 2 provides a brief background on the EnKF and introduces the AEnKF; Section 3 discusses some aspects of application of the AEnKF as well as its relation with the EnKS and the 4D-EnKF; our conclusions are presented in Section 4.

\section{Formulation}

\section{1. $E n K F$}

Let $n$ be the state dimension, $m$ is the ensemble size, and $p$ is the number of observations. Let an $n \times m$ matrix of an ensemble of model states be denoted by $\mathbf{E}$, the ensemble average by $\mathbf{x}$, and the ensemble anomalies by $\mathbf{A}$, so that

$\mathbf{x}=\frac{1}{m} \mathbf{E} \mathbf{1}$,

$\mathbf{A}=\mathbf{E}\left(\mathbf{I}-\frac{1}{m} \mathbf{1 1}^{\mathrm{T}}\right)$

where $\mathbf{1}$ is a vector with all elements equal to one, $\mathbf{I}$ is the identity matrix, and superscript $\mathrm{T}$ denotes matrix transposition. Let also use superscripts $f$ and $a$ to refer to forecast and analysis variables, correspondingly. Then a linear ensemble update can be written in a generic form as

$\mathbf{x}^{a}-\mathbf{x}^{f} \equiv \delta \mathbf{x}=\mathbf{A}^{f} \mathbf{G} \mathbf{s}$,

$\mathbf{A}^{a}-\mathbf{A}^{f} \equiv \delta \mathbf{A}=\mathbf{A}^{f} \mathbf{T}$,

where $\mathbf{s}$ is the scaled innovation vector,

$\mathbf{S}=\mathbf{R}^{-1 / 2}\left(\mathbf{d}-\mathbf{H} \mathbf{x}^{f}\right) / \sqrt{m-1}$,

where $\mathbf{d}$ is the vector of observations, $\mathbf{R}$ is the observation error covariance, and $\mathbf{H}$ is a $p \times n$ matrix of interpolation coefficients from state to observation. For simplicity, we assume linear observations. The scaling of innovation $\mathbf{d}-\mathbf{H} \mathbf{x}^{f}$ is introduced to simplify expressions for the matrix $\mathbf{G}$ introduced in (3).

It is convenient to represent matrices $\mathbf{G}$ and $\mathbf{T}$ in (3) and (4) in terms of scaled ensemble observation anomalies $\mathbf{S}$,

$\mathbf{S} \equiv \mathbf{R}^{-1 / 2} \mathbf{H A}^{f} / \sqrt{m-1}$.

In the EnKF the update (3) of the ensemble mean is independent of the particular scheme used; the matrix $\mathbf{G}$ can be conveniently written as

$$
\begin{aligned}
\mathbf{G} & =\left(\mathbf{I}+\mathbf{S}^{\mathrm{T}} \mathbf{S}\right)^{-1} \mathbf{S}^{\mathrm{T}} \\
& =\mathbf{S}^{\mathrm{T}}\left(\mathbf{I}+\mathbf{S} \mathbf{S}^{\mathrm{T}}\right)^{-1} .
\end{aligned}
$$

Either (7a) or (7b) can be used, depending on the relation between the ensemble size $m$ and the number of observations $p$ : (7a) requires inversion of an $m \times m$ matrix, while (7b) requires inversion of a $p \times p$ matrix. (The idea of performing the inversion in the expression for the Kalman gain in the ensemble space can probably be attributed to Pham et al., 1998; François Counillon, personal communication 2009).

Unlike updating the mean, expressions for the update of ensemble anomalies depend on the scheme used. The two common choices are the traditional EnKF with perturbed observations (Burgers et al., 1998) and the ensemble transform Kalman filter (ETKF; Bishop et al., 2001) that is a flavour of the ensemble square root filter (ESRF). The 'deterministic EnKF' (DEnKF; Sakov and Oke, 2008) can also be a good practical choice (Sun et al., 2009). (The DEnKF represents a suboptimal scheme that is asymptotically equivalent to the ESRF in the case of small corrections.) The expressions for the transform matrix $\mathbf{T}$ for each of the schemes are as follows:

(1) Traditional EnKF:

$\mathbf{T}=\mathbf{G}(\mathbf{D}-\mathbf{S})$,

where $\mathbf{D}=\mathbf{R}^{-1 / 2} \tilde{\mathbf{D}} / \sqrt{m-1}$ is the scaled matrix of random perturbations sampled from a Gaussian distribution so that $\tilde{\mathbf{D}} \mathbf{1}=$ 0 and $\tilde{\mathbf{D}} \tilde{\mathbf{D}}^{\mathrm{T}} /(m-1) \rightarrow \mathbf{R}, \quad m \rightarrow \infty$.

(2) ETKF:

$\mathbf{T}=\left(\mathbf{I}+\mathbf{S}^{\mathrm{T}} \mathbf{S}\right)^{-1 / 2}-\mathbf{I}$.

The notation $(\cdot)^{-1 / 2}$ denotes the inverse of the unique positive definite square root of a positive definite matrix. If the SVD 
decomposition is used for raising $\left(\mathbf{I}+\mathbf{S}^{\mathrm{T}} \mathbf{S}\right)$ to the power of $-1 / 2$ in (9), then it is possible to calculate $\mathbf{G}$ cheaply by using the same decomposition for the inversion in (7a).

(3) DEnKF:

$\mathbf{T}=-\frac{1}{2} \mathbf{G S}$.

It follows from (5) to (10) that to calculate the ensemble update coefficients $\mathbf{G s}$ in (3) and $\mathbf{T}$ in (4), one only needs to know the observations $\mathbf{d}$, the observation error covariance $\mathbf{R}$, and the ensemble observations $\mathbf{H E}^{f}$; and one does not need the full ensemble $\mathbf{E}^{f}$. This result simplifies implementation of the EnKF in large-scale systems, when loading of the ensemble to random access memory is not possible.

\subsection{Asynchronous EnKF}

Consider immediate assimilation of observations $\mathbf{d}_{0}$ made at time $t_{0}$. According to (3) and (4), the assimilation will result in corrections to the ensemble mean and ensemble anomalies that can be written, correspondingly, as a linear combination or a linear transform of the forecast ensemble anomalies

$\delta \mathbf{x}_{0}=\mathbf{A}_{0}^{f} \mathbf{G}_{0} \mathbf{s}_{0}$,

$\delta \mathbf{A}_{0}=\mathbf{A}_{0}^{f} \mathbf{T}_{0}$,

where the subscript 0 is used to tag variables calculated or obtained at time $t_{0}$.

Let us now consider the evolution of the corrections (11) and (12) from time $t_{0}$ to time $t_{1}$, assuming that the model is perfect. Let $\mathbf{M}_{01}$ be the tangent linear propagator for the forecast system trajectory from time $t_{0}$ to time $t_{1}$

$\delta \mathbf{x}_{1}=\mathbf{M}_{01} \delta \mathbf{x}_{0}+O\left(\left\|\delta \mathbf{x}_{0}\right\|^{2}\right)$,

where $\delta \mathbf{x}_{0}$ is an arbitrary perturbation of the state at time $t_{0}, \delta \mathbf{x}_{1}$ is the corresponding perturbation at time $t_{1}$ and $\|\cdot\|$ denotes a norm. Then at time $t_{1}$, in the linear approximation, the corrections (11) and (12) become

$\delta \mathbf{x}_{1} \sim \mathbf{M}_{01} \delta \mathbf{x}_{0}=\mathbf{M}_{01} \mathbf{A}_{0}^{f} \mathbf{G}_{0} \mathbf{s}_{0} \sim \mathbf{A}_{1}^{f} \mathbf{G}_{0} \mathbf{s}_{0}$,

$\delta \mathbf{A}_{1} \sim \mathbf{M}_{01} \delta \mathbf{A}_{0}=\mathbf{M}_{01} \mathbf{A}_{0}^{f} \mathbf{T}_{0} \sim \mathbf{A}_{1}^{f} \mathbf{T}_{0}$,

where $\sim$ denotes 'asymptotically equal', and the subscript 1 is used to tag variables at time $t_{1}$.

Comparing (14) and (15) with (11) and (12), correspondingly, we see that as long as the evolution of the corrections and the ensemble anomalies is linear, the expansion coefficients of the corrections in the basis of ensemble anomalies remain constant along the forecast system trajectory. Normally, in the EnKF the corrections of the ensemble members are of order of the ensemble spread. Consequently, it is normally sufficient for a data assimilation system to be linear in the sense (14) and (15) if the evolution of ensemble anomalies between observation time and assimilation time can be considered linear. In this case the transform matrices calculated for assimilation at the time of observation $t_{0}$ can be used for assimilation at other time $t_{1}$ (Evensen, 2003, 2007). Because the transform matrices are calculated from the ensemble observations, using transform matrices calculated at the time of observation is equivalent to using ensemble observations from that time.

These simple considerations form the basis for the AEnKF. It represents a generic modification of the EnKF and does not rely on a particular analysis scheme. Its feasibility for large-scale applications is based on the fact that calculation of $\mathbf{G}_{0} \mathbf{s}_{0}$ in (14) and $\mathbf{T}_{0}$ in (15) for updating the ensemble at time $t_{1}$ other than the time of observation $t_{0}$ requires only knowledge of ensemble observations $\mathbf{H E}_{0}$ at the time of observation rather than the full ensemble $\mathbf{E}_{0}$.

The AEnKF makes it possible to 'move' the assimilation time of observations without affecting the result. Specifically, at the end of the assimilation window the ensemble mean and the state error covariance carried by the ensemble will be the same for the AEnKF and for the synchronous EnKF updating at each observation time. Because, in the case of a perfect linear model, a full-rank and properly initialized ESRF is equivalent to the Kalman filter, the asynchronous ESRF is in this case equivalent to the Kalman smoother.

We note that, similar to a weak non-linearity, a weak additive model noise does not instantly invalidate the above framework, as long as it does not essentially affect the propagation of corrections and ensemble anomalies, so that the tangent linear hypothesis (13) remains true within the assimilation window.

\subsection{Parallel assimilation of asynchronous observations}

It is known that the result of assimilation of observations with non-correlated error, in terms of the analysed mean and covariance, does not depend on whether the observations are assimilated simultaneously (in parallel) or one by one (serially) (e.g. Bierman, 1977). Because, as discussed above, for a perfect linear model each observation or a group of observations collected at the same time can be assimilated at a different time, provided that the ensemble observations from the time of the observation are used, all $k$ groups of observations collected at times $t_{1}, \ldots$, $t_{k}$ over the length of the assimilation window can be assimilated simultaneously by combining the ensemble observations collected during the assimilation cycle

$\mathbf{H E}=\left[(\mathbf{H E})_{1}^{\mathrm{T}}, \ldots,(\mathbf{H E})_{k}^{\mathrm{T}}\right]^{\mathrm{T}}$,

where the subscripts $1, \ldots, k$ are used to denote ensemble observations at time $t_{1}, \ldots, t_{k}$. Note that collecting ensemble observations (16) within an assimilation window involves integrating the forecast ensemble to the end of the window.

The above method (16) of concatenating asynchronous ensemble observations has been described in (Hunt et al., 2007, Section 4). Applied for 'local ETKF' (LETKF), it has been referred to as 4D-LETKF. The 4D-LETKF has been studied in 
experiments with the 40-D Lorentz model in Fertig et al. (2007), and with a global circulation model in Harlim and Hunt (2007).

In the case of a perfect linear model one can use a global assimilation window. In this framework using asynchronous ensemble observations (16) is equivalent to the ensemble smoother solution (van Leeuwen and Evensen, 1996, eqs 38-40).

The relative impact of observations collected at different times depends, with all other factors being equal, on the magnitude of the scaled ensemble observation anomalies $\left(\mathbf{R}^{-1 / 2} \mathbf{H A}^{f}\right)_{i}$ at each particular time $t_{i}$. As the forecast ensemble anomalies, generally, tend to grow with time, with all other factors being equal, later observations do have a bigger impact on the analysis than earlier observations.

\section{Discussion}

\subsection{EnKS}

Using asynchronous ensemble observations (16) is equivalent to applying corresponding ensemble transform matrices, as in the EnKS solution (Evensen, 2003, eq. 105)

$\mathbf{E}^{a}=\mathbf{E}^{f} \prod_{i=1}^{k} \mathbf{X}_{5}\left(t_{i}\right)$,

where $\mathbf{X}_{5}\left(t_{i}\right)$ is the ensemble transform matrix for assimilation of observations at time $t_{i}$; it can be represented in terms of $\mathbf{G}, \mathbf{s}$ and $\mathbf{T}$ introduced in (3) and (4) as follows:

$\mathbf{X}_{5}=\frac{1}{m} \mathbf{1 1}^{\mathrm{T}}+\left(\mathbf{I}-\frac{1}{m} \mathbf{1 1}^{\mathrm{T}}\right)\left(\mathbf{G} \mathbf{s} \mathbf{1}^{\mathrm{T}}+\mathbf{T}\right)$.

Note however that the ensemble transform matrices $\mathbf{X}_{5}\left(t_{i}\right)$ must be calculated sequentially, so that the transform matrix for each next group of observations is calculated using analysed ensemble from assimilating all previous groups. Therefore, eq. (17) implicitly involves an ensemble update for each group of observations, while combining observations by (16) makes it possible to assimilate all of them in a single ensemble update. One can see (17) as equivalent to assimilating observations in batches (formed in this case according to the observation time), which requires an ensemble update after assimilating each batch.

\subsection{Joint state-observation space}

The AEnKF solution (16) is equivalent to the concept of joint state-observation space (Tarantola, 1987; Brian Hunt, personal communication 2009): if one formally replaces the ensemble $\mathbf{E}$ by the joint ensemble $\tilde{\mathbf{E}}$,

$\tilde{\mathbf{E}}=\left[\mathbf{E}^{\mathrm{T}},(\mathbf{H E})_{1}^{\mathrm{T}}, \ldots,(\mathbf{H E})_{k}^{\mathrm{T}}\right]^{\mathrm{T}}$,

then updating this joint ensemble in the EnKF framework is equivalent to using combined asynchronous ensemble observations (16). In the context of Section 2.2 this equivalence means that the concept of joint state-observation space is applicable in situations when the update is made at the time different to the time of observations if evolution of corrections and ensemble anomalies along the forecast trajectory is described by a linear propagator.

\subsection{Localization}

There currently exist two common localization methods for the EnKF. The first method modifies the Kalman gain by replacing the state error covariance by its element-wise product with some distance-dependent correlation matrix (Houtekamer and Mitchell, 2001; Hamill and Whitaker, 2001). It is commonly referred to as covariance localization, or covariance filtering. The second method uses a local approximation of the forecast covariance for updating a state vector element, calculated by building a local window around this element (Anderson, 2003; Evensen, 2003; Ott et al., 2004). Following Evensen (2003), we will refer to it as local analysis.

The local analysis can be conducted by building for each updated state vector element a local set of ensemble observations from the global set. Technically it can be used with the AEnKF in the same way as with the EnKF, provided that the asynchronous ensemble observations are used.

The covariance localization involves calculation of products $\left(\boldsymbol{\rho} \circ \mathbf{P}^{f}\right) \mathbf{H}^{\mathrm{T}}$ and $\mathbf{H}\left(\boldsymbol{\rho} \circ \mathbf{P}^{f}\right) \mathbf{H}^{\mathrm{T}}$, where $\rho$ is a so-called correlation matrix, and $\circ$ denotes element-wise product of matrices. In practice these two products are usually replaced by

$\left(\boldsymbol{\rho} \circ \mathbf{P}^{f}\right) \mathbf{H}^{\mathrm{T}} \rightarrow \sum_{o=1}^{p}\left(\stackrel{o}{\boldsymbol{\rho}} \circ \mathbf{A}^{f}\right)\left(\stackrel{o}{\mathbf{H}} \mathbf{A}^{f}\right)^{\mathrm{T}}$,

$\mathbf{H}\left(\boldsymbol{\rho} \circ \mathbf{P}^{f}\right) \mathbf{H}^{\mathrm{T}} \rightarrow\left(\mathbf{H} \rho \mathbf{H}^{\mathrm{T}}\right) \circ\left[\left(\mathbf{H A}^{f}\right)\left(\mathbf{H A}^{f}\right)^{\mathrm{T}}\right]$,

where $\rho$ is the matrix of $m$ identical column vectors equal to the oth column of $\rho \mathbf{H}^{\mathrm{T}}$ and $\stackrel{o}{\mathbf{H}}$ denotes $\mathbf{H}$ with all rows but the $o$ th set to zero. To adapt these expressions to the AEnKF, one just needs to replace the forecast ensemble observation anomalies by the asynchronous ensemble observation anomalies (Brian Hunt, personal communication 2009):

$\mathbf{H A}^{f} \rightarrow \mathbf{H A}=\left[(\mathbf{H A})_{1}^{\mathrm{T}}, \ldots,(\mathbf{H A})_{k}^{\mathrm{T}}\right]^{\mathrm{T}}$.

\section{4. $4 D-E n K F$}

Hunt et al. (2004) proposed an extension of the EnKF for assimilation of asynchronous data called 4D-EnKF. In the 4D-EnKF, to assimilate at time $t_{1}$ observations $\mathbf{d}_{0}$ collected at time $t_{0}$, the ensemble mean $\mathbf{x}_{0}$ and ensemble anomalies $\mathbf{A}_{0}$ are replaced as follows:

$$
\begin{aligned}
& \mathbf{H x}_{0}^{f} \rightarrow \mathbf{H E}_{0}\left(\mathbf{E}_{1}^{\mathrm{T}} \mathbf{E}_{1}\right)^{-1} \mathbf{E}_{1}^{\mathrm{T}} \mathbf{x}_{1}^{f}, \\
& \mathbf{H A}_{0}^{f} \rightarrow \mathbf{H E}_{0}\left(\mathbf{E}_{1}^{\mathrm{T}} \mathbf{E}_{1}\right)^{-1} \mathbf{E}_{1}^{\mathrm{T}} \mathbf{A}_{1}^{f} .
\end{aligned}
$$


(For readability we omit superscripts $f$ for the forecast ensemble matrices $\mathbf{E}_{0}^{f}$ and $\mathbf{E}_{1}^{f}$.)

If $m \leq n$ and $\mathbf{E}$ has full rank, then the product $\mathbf{E}^{\mathrm{T}} \mathbf{E}$ is nonsingular, and $\left(\mathbf{E}^{\mathrm{T}} \mathbf{E}\right)^{-1} \mathbf{E x}$, where $\mathbf{x}$ is an arbitrary vector, represents a vector of expansion coefficients in the basis of $\mathbf{E}$ of the orthogonal projection of $\mathbf{x}$ onto the range of $\mathbf{E}$. Because $\mathbf{x}_{1}^{f}$ in (19a) is the ensemble average, $\left(\mathbf{E}_{1}^{\mathrm{T}} \mathbf{E}_{1}\right)^{-1} \mathbf{E}_{1}^{\mathrm{T}} \mathbf{x}_{1}^{f}=\mathbf{1} / \mathrm{m}$, and $\mathbf{E}_{0}\left(\mathbf{E}_{1}^{\mathrm{T}} \mathbf{E}_{1}\right)^{-1} \mathbf{E}_{1}^{\mathrm{T}} \mathbf{x}_{1}^{f}=\mathbf{x}_{0}^{f}$, so that the replacement (19a) has no effect (is an identity). Similarly, $\left(\mathbf{E}_{1}^{\mathrm{T}} \mathbf{E}_{1}\right)^{-1} \mathbf{E}_{1}^{\mathrm{T}} \mathbf{A}_{1}^{f}=\mathbf{I}-\mathbf{1 1}^{\mathrm{T}} / m$, $\mathbf{E}_{0}^{f}\left(\mathbf{E}_{1}^{\mathrm{T}} \mathbf{E}_{1}\right)^{-1} \mathbf{E}_{1}^{\mathrm{T}} \mathbf{A}_{1}^{f}=\mathbf{A}_{0}^{f}$, and (19b) also has no effect. If $m>n$ or $\mathbf{E}$ is rank deficient, then replacements (19) become singular.

Therefore, in the case when the ensemble size is not greater than the state dimension and the ensemble has full rank, the 4D-EnKF is equivalent to the AEnKF; otherwise it becomes singular.

The equivalence between the AEnKF and 4D-EnKF has been recognized in a number of studies. Harlim and Hunt (2007) argued the AEnKF (4D-LETKF) is 'mathematically equivalent' to $4 \mathrm{D}$-EnKF but 'computationally more efficient since the analysis needs no calculations of eigenvectors'. In Fertig et al. (2007) the AEnKF (4D-LETKF) has been characterized as a 'simplified version of the 4D-EnKF in Hunt et al. (2004)'.

\subsection{Conditions of applicability}

Below we briefly list a number of factors that affect the applicability of the AEnKF, that is the possible length of the assimilation window during asynchronous data assimilation with the EnKF. Generically, these factors can be considered as different kinds of suboptimality of the system; and the system becomes progressively more vulnerable in regard to suboptimalities as the length of the assimilation window increases.

(1) Non-linearity: The non-linearity of the system represents a combined effect of the non-linearity of the model or the observation operator, and the finite nature of the ensemble perturbations about the ensemble mean; so that either a less nonlinear or a better constrained model allow a longer assimilation window.

(2) Model error: In this study, we assumed the perfect model scenario that corresponds to the traditional 'strong-constraint' four-dimensional variational data assimilation (4D-Var). In practice it can be expected that because of the model error the propagation of corrections along the trajectory can become increasingly noisy as the assimilation window becomes longer.

(3) Localization: The localization is unavoidable in largescale systems. It involves a local approximation of the underlying Kalman filter framework, and therefore contributes towards the suboptimality of the system.

(4) Suboptimal scheme: Some analysis schemes, such as the DEnKF, may involve approximations to the Kalman filter. It can be expected in these cases that a data assimilation system will show progressively deteriorating performance for longer assimilation windows compared to systems with optimal schemes.

\section{Conclusions}

This study revisited the problem of assimilation of asynchronous observations with the EnKF. We showed that a modification of the EnKF that we refer to as the asynchronous EnKF, or AEnKF, makes it possible to assimilate asynchronous observations.

We came to the AEnKF by using the concept of propagation of corrections along the forecast system trajectory, assuming a perfect model and linear propagation of ensemble anomalies within the assimilation window. The AEnKF itself involves calculating update using ensemble observations from the time of observations (asynchronous ensemble observations), rather than from the time of update, as in the synchronous EnKF, when all assimilated observations are assumed to be synchronous. This modification to the EnKF is so simple that we suggest using it in EnKF systems on a routine basis.

The AEnKF is formally equivalent to the EnKS solution (17) written in the form of updating the ensemble via a product of transform matrices from the forward run of the EnKF (Evensen, 2003); however, it may be more suitable for use in large-scale systems because, unlike that form of the EnKS solution, it does not require multiple ensemble updates.

There is an extensive literature on theoretical equivalence of 4D-Var and Kalman smoother. 'For an imperfect linear model, weak-constraint 4D-Var is equivalent to a fixed-interval Kalman smoother initialized with the same background state and covariance matrix at the beginning of the assimilation window' (Fisher et al., 2005). Because, as argued in Section 2.2, in the case of perfect linear model the asynchronous ESRF is equivalent to the Kalman smoother, the AEnKF in this case is theoretically equivalent to strong-constraint 4D-Var, but does not require a tangent linear or adjoint model.

We underline that although an idealized framework was used for introducing the AEnKF that included assumptions of linear observations and a perfect linear model, similarly to the EnKF, the AEnKF can be used in practice as a suboptimal solution in situations when these assumptions are weakly violated.

The 4D-EnKF method developed by Hunt et al. (2004) is normally equivalent to the EnKS solution used in the AEnKF, but involves unnecessary additional calculations and can become singular in some situations.

\section{Acknowledgments}

The authors would like to thank Brian Hunt, Olwijn Leeuwenburgh and an anonymous reviewer for comments and suggestions that helped to substantially improve the quality of the manuscript. 
The authors gratefully acknowledge funding from the eVITAEnKF project by the Research Council of Norway.

\section{References}

Anderson, J. L. 2003. A local least squares framework for ensemble filtering. Mon. Wea. Rev. 131, 634-642.

Bierman, G. J. 1977. Factorization Methods for Discrete Sequential Estimation, Academic Press, New York, 241.

Bishop, C. H., Etherton, B. and Majumdar, S. J. 2001. Adaptive sampling with the ensemble transform Kalman filter. Part I: theoretical aspects. Mon. Wea. Rev. 129, 420-436.

Burgers, G., van Leeuwen, P. J. and Evensen, G. 1998. Analysis scheme in the ensemble Kalman filter. Mon. Wea. Rev. 126, 1719-1724.

Evensen, G. 1994. Sequential data assimilation with a nonlinear quasigeostrophic model using Monte-Carlo methods to forecast error statistic. J. Geophys. Res. 99, 10143-10162.

Evensen, G. 2003. The ensemble Kalman filter: theoretical formulation and practical implementation. Ocean Dyn. 53, 343-367.

Evensen, G. 2007. Data Assimilation: The Ensemble Kalman Filter, Springer, Berlin, 279.

Evensen, G. and van Leeuwen, P. J. 2000. An ensemble Kalman smoother for nonlinear dynamics. Mon. Wea. Rev. 128, 1852-1867.

Fertig, E. J., Harlim, J. and Hunt, B. R. 2007. A comparative study of 4DVAR and a 4D Ensemble Kalman Filter: perfect model simulations with Lorenz-96. Tellus 59A, 96-100.

Fisher, M., Leutbecher, M. and Kelly, G. A. 2005. On the equivalence between Kalman smoothing and weak-constraint four-dimensional variational data assimilation. Quart. J. Roy. Meteor. Soc. 131, 32353246.
Hamill, T. M. and Whitaker, J. S. 2001. Distance-dependent filtering of background error covariance estimates in an ensemble Kalman filter. Mon. Wea. Rev. 129, 2776-2790.

Harlim, J. and Hunt, B. R. 2007. Four-dimensional local ensemble transform Kalman filter: numerical experiments with a global circulation model. Tellus 59A, 731-748.

Houtekamer, P. L. and Mitchell, H. L. 2001. A sequential ensemble Kalman Filter for atmospheric data assimilation. Mon. Wea. Rev. 129, 123-137.

Hunt, B. R., Kalnay, E., Kostelich, E. J., Ott, E., Patil, D. J, and coauthors. 2004. Four-dimensional ensemble Kalman filtering. Tellus 56A, 273-277.

Hunt, B. R., Kostelich, E. J. and Szunyogh, I. 2007. Efficient data assimilation for spatiotemporal chaos: a local ensemble transform Kalman filter. Physica D 230, 112-126.

Ott, E., Hunt, B. R., Szunyogh, I., Zimin, A. V., Kostelich, E. J. and coauthors. 2004. A local ensemble Kalman filter for atmospheric data assimilation. Tellus 56A, 415-428.

Pham, D. T., Verron, J. and Roubaud, M. C. 1998. A singular evolutive extended Kalman filter for data assimilation in oceanography. J. Mar. Syst. 16, 323-340.

Sakov, P. and Oke, P. R. 2008. A deterministic formulation of the ensemble Kalman filter: an alternative to ensemble square root filters. Tellus 60A, 361-371.

Sun, A. Y., Morris, A. and Mohanty, S. 2009. Comparison of deterministic ensemble Kalman filters for assimilating hydrogeological data. Adv. Water Resour. 32, 280-292.

Tarantola, A. 1987. Inverse Problem Theory, Elsevier, New York, 613. van Leeuwen, P. J. and Evensen, G. 1996. Data assimilation and inverse methods in terms of a probabilistic formulation. Mon. Wea. Rev. 124, 2898-2913. 